\section{HIV-Therapie 2014 - State of the Art}

\author{
G. Fätkenheuer ${ }^{1}$ \\ ${ }^{1}$ Leiter Infektiologie, Klinik I für Innere Medizin, Universitätsklini- \\ kum Köln (AöR)
}

Nach den Angaben der WHO lebten im Jahr 201235 Millionen Menschen mit einer HIV-Infektion, und 2,3 Millionen Personen infizierten sich neu mit HIV. Weltweit befanden sich im Jahr 2012 9,7 Millionen Menschen in Regionen mit niedrigem und mittlerem Einkommen unter antiretroviraler Therapie. Das entspricht $61 \%$ der Patienten, die nach den Behandlungsempfehlungen der WHO von 2010 eine antiretrovirale Therapie benötigen. Das Ziel der WHO ist es, dass bis 201515 Millionen HIV-infizierte Menschen antiretroviral behandelt werden [1]. Für Deutschland geht das Robert-KochInstitut davon aus, dass es im Jahr 20133263 gesicherte HIV-Neudiagnosen gab und damit 10\% mehr als im Jahr zuvor. In Deutschland und in anderen entwickelten Ländern haben HIV-Patienten unter einer lebenslangen Therapie inzwischen eine weitgehend normale Lebenserwartung. Todesfälle durch HIV-assoziierte Erkrankungen sind selten geworden, dafür wird die Sterblichkeit von HIV-infizierten Patienten durch andere Erkrankungen, allen voran Krebserkrankungen, angeführt [2].

Für die Langzeitbehandlung von HIV-infizierten Patienten stehen inzwischen mehr als 20 einzelne Substanzen zur Verfügung, die in der Regel als Kombination von drei wirksamen Präparaten verabreicht werden. Im einfachsten Falle erfolgt eine Kombinationstherapie mit einer Tablette pro Tag [3]. Inzwischen gibt es in allen Substanzklassen mehrere Medikamente zur Auswahl. Insbesondere in der Klasse der Integrasehemmer gab es in den beiden letzten Jahren Neuzulassungen in Europa (Elvitegravir, Dolutegravir), mit denen die Behandlungsmöglichkeiten nochmals erweitert wurden. Dolutegravir wurde 2014 in Deutschland eingeführt. In klinischen Studien bei unterschiedlichsten Patienten und mit verschiedenen Vergleichsregimen hat die Substanz in Kombination mit zwei Nukleosidanaloga sehr gute Ergebnisse erbracht [4]. Eine weitere Neueinführung ist Cobicistat, das als pharmakologische Boostersubstanz eine Alternative zu Ritonavir darstellt. Für die Initialtherapie wird in den verschiedenen nationalen und internationalen Leitlinien eine Kombinationstherapie mit zwei Nukleosidanaloga und entweder einem nicht nukleosidischen Reversetranskriptasehemmer, einem geboosterten Proteasehemmer oder einem Integrasehemmer empfohlen. Die aktuellen Leitlinien der DeutschÖsterreichischen Aidsgesellschaft (DAIG) vom Mai 2014 ermöglichen eine große Zahl von Kombinationen mit den aufgeführten Substanzklassen [5].

Unterschiede in den Therapieempfehlungen gibt es vor allem zur Frage des Therapiestarts. Die amerikanischen Leitlinien empfehlen eine antiretrovirale Therapie (ART) für alle HIVinfizierten Patienten, unabhängig vom klinischen Status und von der Zahl der CD4-Zellen [6]. Die WHO-Leitlinien sehen eine Therapie für alle Patienten mit einer CD4-Zellzahl von

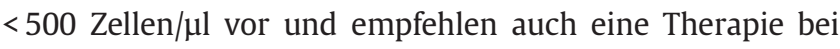

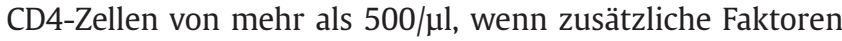
vorliegen (aktive Tuberkulose, chronische Hepatitis B, Schwangerschaft oder serodiskordante Partnerschaft) [7]. Dagegen empfehlen die europäischen und die deutsch-österreichischen Leitlinien eine ART bei asymptomatischen Patienten erst dann zwingend, wenn die CD4-Zellen unter 350/

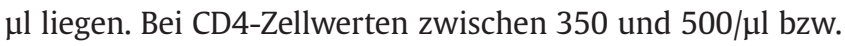

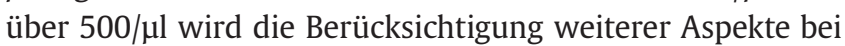
der Entscheidung zur Therapieeinleitung empfohlen [5]. Eine definitive Entscheidung über den besten Zeitpunkt zum Start einer ART wird von der randomisierten, derzeit laufenden START-Studie mit mehr als 4600 Teilnehmern erwartet [8].

Da die lebenslange antiretrovirale Therapie potenziell mit einer Reihe von Nebenwirkungen behaftet und außerdem teuer ist, hat die Suche nach einer definitiven Heilung der Erkrankung in den letzten Jahren starken Auftrieb erhalten. Zwei besondere klinische Fälle waren hieran besonders beteiligt: Zum einen handelte es sich um die Heilung eines an Leukämie erkrankten HIV-Patienten („Berlin Patient“) durch die allogene Transplantation mit Stammzellen eines CCR5-negativen Spenders [9], zum anderen um den Verlauf bei dem sogenannten Mississippi Baby [10]. Im letzteren Fall war ein HIV-infizierter Säugling, der unmittelbar nach der Entbindung antiretroviral behandelt worden war, über einen Zeitraum von mehr als 2 Jahren ohne nachweisbare Viruslast geblieben. Dies wurde als Hinweis darauf gewertet, dass eine sehr frühzeitige ART die Einnistung von HIV in sogenannten latenten Reservoirs verhindern könne. Im Juli 2014 wurde dann jedoch berichtet, dass es bei dem Kind nach langer Latenz erneut zum Virusnachweis im Blut gekommen ist [11]. Diese Beobachtung hat der Euphorie über die rasche Entwicklung einer Heilung von HIV einen Dämpfer gegeben. Zudem ist ein Versuch bei zwei Patienten aus Boston gescheitert, eine Heilung durch allogene Stammzelltransplantation zu erreichen [12]. Die Suche nach einer Heilung steht damit vor einem Neubeginn und vor vielen offenen Fragen [13]. Von entscheidender Bedeutung wird es sein, ob es gelingt, die Verstecke von HIV im Körper besser zu identifizieren mit dem Ziel, auch dort das Virus wirksam zu treffen.

Zusammenfassung: In den entwickelten Ländern bestehen heute sehr gute Möglichkeiten für eine langfristig wirksame Behandlung der HIV-Infektion. Es stehen ausreichend Substanzen zur Verfügung, um praktisch alle Patienten mit gut verträglichen Regimen behandeln zu können. Die derzeitigen Anstrengungen richten sich besonders auf Interventionen, die eine vollständige Heilung der HIV-Infektion ermöglichen. Interessenkonflikte: Forschungsförderung: Gilead, Janssen, Merck Sharp \& Dohme, Pfizer, ViiV Healthcare. Vortragshonorare: Janssen-Cilag, Merck Sharp \& Dohme Advisory Boards: Bristol-Myers Squibb, Gilead, Janssen, Merck Sharp \& Dohme

\section{Literatur}

1 WHO. Global Report - UNAIDS report on the global AIDS epidemic 2013 (2013)

2 Smith CJ, Ryom L, Weber $R$ et al. Trends in underlying causes of death in people with HIV from 1999 to 2011: a multicohort collaboration. Lancet 2014; 384: $241-248$

3 Gandhi M, Gandhi RT. Single-pill combination regimens for treatment of HIV-1 infection. N Engl J Med 2014; 371: 248 - 259 
4 Osterholzer DA, Goldman M. Dolutegravir: a next-generation integrase inhibitor for treatment of HIV infection. Clin Infect Dis 2014; 59: $265-271$

5 Deutsche AIDS Gesellschaft. Deutsch-Österreichische Leitlinien zur antiretroviralen Therapie der HIV-Infektion (s2 k) (2014). Im Internet: www.daignet.de; Stand: Mai 2014

6 Gunthard HF, Aberg JA, Eron IJ et al. Antiretroviral treatment of adult HIV infection: 2014 recommendations of the International Antiviral Society-USA Panel. JAMA 2014; 312: 410-425

7 WHO. The use of antiretroviral drugs for treating and preventing HIV infection (2013)

8 Babiker AG, Emery S, Fatkenheuer G et al. Considerations in the rationale, design and methods of the Strategic Timing of AntiRetroviral Treatment (START) study. Clinical Trials 2013; 10: S5 -S36

9 Hutter G, Nowak D, Mossner M et al. Long-term control of HIV by CCR5 Delta32/Delta32 stem-cell transplantation. N Engl J Med 2009; 360: $692-698$

10 Persaud D, Gay H, Ziemniak C et al. Absence of detectable HIV-1 viremia after treatment cessation in an infant. N Engl J Med 2013; 369: $1828-1835$

11 Siliciano JD, Siliciano RF. AIDS/HIV. Rekindled HIV infection. Science 2014; 345: 1005-1006
12 Henrich TJ, Hanhauser E, Marty FM et al. Antiretroviral-free HIV-1 remission and viral rebound after allogeneic stem cell transplantation: report of 2 cases. Ann Intern Med 2014; 161: 319-327

13 Lewin SR. Finding a cure for HIV: much work to do. Ann Intern Med 2014; $161: 368-369$

Bibliografie

Dol http://dx.doi.org/10.1055/s-0033-1358034

Drug Res 2014; 64, Suppl. 1: S16-S17

(c) Georg Thieme Verlag KG Stuttgart · New York .

ISSN 2194-9379

Korrespondenzadresse

Gerd Fätkenheuer

Universitätsklinikum Köln

Kerpener Straße 62

50937 Köln

gerd.faetkenheuer@uk-koeln.de 